\title{
Study on the carbon fluxes and their influencing factors in a large reservoir
}

\author{
Y.C.WANG ${ }^{1,2}$,G.J.WANG ${ }^{1,3}$, M.M.HU ${ }^{1,2}$
}

${ }^{1}$ State Key Laboratory of Simulation and Regulation of Water Cycle in River Basin, Beijing 10038, China.

${ }^{2}$ Department of water environment, China Institute of water resources and hydropower research, Beijing 10038, China

${ }^{3}$ China Three Gorges University, Yichang 443002, China

The water environment and water ecological of the Three Gorges Reservoir after impoundment have received widespread attention across various scientific communities, and the potential carbon emissions cannot be ignored Although a lot of research about it has been conducted, the results is not enough to understanding the characteristics and mechanism of carbon cycling in the There Gorges Reservoir.Here,we studied the carbon flux across water-air interface in the mainstream and tributaries of the Three Gorges Reservoir with in-situ monitoring and laboratory simulation from 2015-2017. The $\mathrm{CH}_{4}$ flux ranged from 0.036 to $0.248 \mathrm{mg} /\left(\mathrm{m}^{2} \cdot \mathrm{h}\right)$, which is lower than that of other reservoirs. The fluxes of carbon dioxide aross the water-air interface appeared an obvious different characteristic of monthly variation in main stream and tributaries Main stream and CaoTang River performance for "carbon source" of carbon dioxide,and Zhuyi River showed source and sink alternately.The results also indicated that the fluxes of carbon dioxide of CaoTang River across the water-air interface appeared an obvious characteristic of diurnal variation at different water levels. the overall carbon dioxide showed "absorb by day and emit at night" in the initial impoundment period,however emitted all day in dry seasons.Environmental factors such as dissolved oxygen, water tempreture,wind speed, water level and nutrient concentration play a key role in carbon imission.Based the bubble flux of methane, our result also suggested that in summer, the contrubution of bubbles to cabon flux cannot be ignored in some area of tributary. 